\title{
Lernzeiten und Lernräume etablieren
}

\section{Gelingensbedingungen zur Teilnahme an wissenschaftlicher Weiterbildung}

\author{
Ramona Kahl (iD)
}

Eingegangen: 21. Dezember 2018 / Angenommen: 31. Mai 2019 / Online publiziert: 11. Juni 2019

(C) Der/die Autor(en) 2019

Zusammenfassung Der Beitrag setzt sich mit der Frage auseinander, wie das Lernen in der wissenschaftlichen Weiterbildung in zeitlicher und räumlicher Hinsicht gestaltet und ermöglicht wird. Ansatzpunkt der Betrachtung bilden die Präsenzphasen und die Selbstlernphasen der Teilnehmenden, die als zwei Varianten des Umgangs mit Zeit und Raum beim Weiterbildungslernen betrachtet werden. Beide Formate weisen für die Teilnehmenden eigene Herausforderungen auf, wobei deutlich wird, dass die Qualität der Lernzeit und des Lernorts eine zentrale Rolle spielt. Zudem sind Zeiten und Räume für das Präsenz- und das Selbststudium sozial zu legitimieren. Der Erfolg der Etablierungsbemühungen wird von der Flexibilität der Teilnehmenden und ihrer beruflichen und privaten Kontexte bedingt.

Schlüsselwörter Lernzeit · Lernraum · Präsenzstudium · Selbststudium · Wissenschaftliche Weiterbildung · Teilnehmende

\section{Establishing learning times and learning spaces}

Conditions for a successful participation in university continuing education

\begin{abstract}
The article deals with the question how learning is made possible in time and space in university continuing education. The analysis focuses on the phases of on-site study and self-study, which are regarded as two forms of dealing with time and space in university continuing education. Both formats present specific challenges for the students, showing that the quality of learning time and learning spaces plays a key role in participation. In addition, times and places for on-site study and self-study have to be socially legitimized and established. The success of
\end{abstract}

Dr. R. Kahl (四)

Philipps-Universität Marburg, Marburg, Deutschland

E-Mail: kahl@uni-marburg.de 
these efforts depends on the flexibility of the participants and their professional and private contexts.

Keywords Learning time $\cdot$ Learning space $\cdot$ On-site study $\cdot$ Self-study $\cdot$ University continuing education $\cdot$ Students

\section{Einführung}

Wissenschaftliche Weiterbildung stellt neben Lehre und Forschung eine weitere Säule im Aufgabenportfolio der Hochschulen dar. Trotz ihrer zentralen gesetzlichen Stellung nehmen wissenschaftliche Weiterbildungsangebote an Hochschulen erst in den letzten Jahren zu. Die Zurückhaltung in der Erfüllung dieser Aufgabe rührt unter anderem von der „fremden“ organisationalen Logik der wissenschaftlichen Weiterbildung im Vergleich zum grundständigen Studium her. ${ }^{1}$ Als ,,spezifisches Angebotssegment berufsbegleitender, abschlussorientierter und kostenpflichtiger Weiterbildung für individuelle und institutionelle Zielgruppen“ (Seitter 2017a, S. 146) bilden Bedarfserschließung, Vollkostenfinanzierung, Dienstleistungs- und Nachfrageorientierung maßgebliche Eckpfeiler solcher Hochschulangebote. Zu ihren Kernmerkmalen zählt ebenfalls, dass sie sich vor allem an erwerbstätige Erwachsene richtet, die eine heterogene Zielgruppe hinsichtlich Berufserfahrung, Familienstand, Lebensalter, Lernbiographie sowie fachkultureller Prägung darstellen (vgl. Jütte und Bade-Becker 2018, S. 826f.; Hanft und Knust 2010, S. 49f.). Bei aller Diversität zeichnet sich die Teilnahme durch eine grundlegende Gemeinsamkeit aus: begrenzte Zeit. $^{2}$

Auf der individuellen Ebene ist das durch die vielfältigen Verpflichtungen erwerbstätiger Erwachsener im beruflichen, familiären und soziokulturellen Bereich bedingt. Dadurch ist die Weiterbildungszielgruppe terminlich weniger flexibel und kann weniger frei über ihre (Lern-)Zeiten und Lernorte verfügen. Auf der strukturellen Ebene stehen derzeit keine eigenständigen Zeitkontingente für Weiterbildung zur Verfügung. „Durch die arbeitsrechtliche Zweiteilung der Zeit - entweder Arbeitsoder Freizeit - gerät Lernen in die Klemme“ (Faulstich 2003, S. 17). Infolgedessen sind ,,individuelle Anstrengungen, organisatorische Aufwendungen [...] (und) öffentliche Förderung“ (Faulstich 2005, S. 214) notwendig, um (Zeit)Ressourcen für Weiterbildung zu eröffnen. Aufgrund dieser Rahmenbedingungen erweist sich Zeit „als (individuelle) Weiterbildungsbarriere“ (Schmidt-Lauff 2008, S. 237). Dies gilt auch für die wissenschaftliche Weiterbildung an Hochschulen, die ,als berufs- oder familienbegleitendes didaktisches Arrangement [...] Zeitknappheit als Ausgangsprämisse (hat)“" (Seitter 2017b, S. 11).

Auf die Zeitressource nehmen zudem räumliche Faktoren Einfluss. Das fängt bei der Bedeutung des Standorts der Weiterbildung und möglichen Fahrtzeiten an und reicht bis zur räumlichen Gestaltung von Lehr-Lern-Settings und selbstorganisierten

\footnotetext{
${ }^{1}$ Im Verhältnis zur allgemeinen und betrieblichen Weiterbildung ,,(bildet) ihre ,Wissenschaftlichkeit“ [...] das Alleinstellungsmerkmal der Weiterbildung an Hochschulen“ (Jütte und Bade-Becker 2018, S. 828).

2 Siehe Adult Education Survey der Europäische Union (vgl. CEDEFOOP 2012, S. 7).
} 
Lernumgebungen. Eine Umgebung wird zu einem Lernraum, ,wenn die Konstellation von Wissensträger, Infrastruktur, Atmosphäre und Ko-Präsenz für eine bestimmte Person und einen konkreten Lerngegenstand zu einem gegebenen Zeitpunkt passend ist“ (Kraus 2015, S. 49). ${ }^{3}$ Somit lässt sich im Anschluss an Schmidt-Lauff „Lernen als Handeln in der Zeit" (2012, S. 12) und im Raum definieren. Auf dieser Basis wird in den folgenden Ausführungen unter Lernzeit der temporale Aufwand zur Teilnahme an wissenschaftlicher Weiterbildung - dem Besuch der Lehrveranstaltungen wie der Auseinandersetzung und Aneignung der Bildungsinhalte - verstanden. Als Lernorte gelten mit Kraus (ebd.) räumliche Arrangements von personellen, infrastrukturellen, atmosphärischen und inhaltlichen Elementen. Dabei kann es sich sowohl um institutionell bereitgestellte als auch individuell hergestellte Lernräume handeln. ${ }^{4}$

Diverse Studien im Feld der wissenschaftlichen Weiterbildung haben die Bedeutung des Faktors Zeit für die passfähige Angebotsgestaltung und erfolgreiche Teilnahme benannt (vgl. Präßler 2015, S. 175, 178 f.; Kahl et al. 2015, S. 330f.; 346; Wonneberger et al. 2015, S. 78; Seitter 2017b). Ebenso werden darin geeignete Lernorte und räumliche Bedingungen als relevante Gestaltungsfaktoren ausgewiesen (Kahl et al. 2015, S. 346f.; Rumpf und Salland 2017, S. 154f.). Doch einschlägige Forschungen zu Lernorten und Lernzeiten im Kontext der Hochschulbildung finden sich bislang nur für das grundständige Studium (vgl. Schulmeister und Metzger 2011; Vogel und Woisch 2013,) und das Fernstudium (vgl. Thuy und Höllermann 2011; Sommerfeldt und Höllermann 2014).

Angesichts dessen ist im Rahmen des Verbundprojekts „,WM ${ }^{3}$ Weiterbildung Mittelhessen“ das Forschungsprojekt "Individuumsbezogene Zeitbudgetstudie“ (2015-2017) durchgeführt worden. ${ }^{5}$ Die Studie hat zeitliche Vereinbarkeitsstrategien und Lernzeitbudgets von Teilnehmenden hochschulischer Weiterbildungsangebote unter Berücksichtigung von familiären, sozialen und beruflichen Bedingungen untersucht. Der vorliegende Beitrag basiert auf den empirischen Befunden der „Individuumsbezogenen Zeitbudgetstudie“ und nimmt eine Re-Analyse des Interviewmaterials bezüglich der möglichen Zeiten und Räume des Lernens aus Teilnehmendenperspektive vor. Im Schwerpunkt setzt er sich mit der Frage auseinander, wie das Lernen in der wissenschaftlichen Weiterbildung in zeitlicher und räumlicher Hinsicht gestaltet und ermöglicht wird. Dies erfolgt zentral entlang der Differenzierung in die beiden didaktischen Lehr-Lern-Formate Präsenzstudium

\footnotetext{
3 Im Beitrag wird der Begriff „Lernraum“ statt „Lernort“ verwendet, da er die „Aneignung eines Ortes im Vollzug des Lernens [...] betont“" (Stang et al. 2018, S. 648).

4 Kraus spricht diesbezüglich von pädagogisch gestalteten und selbstgestalteten Lernorten (2015, S. 49).

5 Die Justus-Liebig-Universität Gießen, Philipps-Universität Marburg und Technische Hochschule Mittelhessen haben sich mittels des BMBF-Wettbewerbs „Aufstieg durch Bildung: offene Hochschulen“ zum Verbundprojekt „WM ${ }^{3}$ Weiterbildung Mittelhessen“ zusammengeschlossen, um wissenschaftliche Weiterbildungsangebote an ihren Hochschulen zu stärken. Das Projekt ist in der ersten Förderphase (2011-2015) aus Mitteln des BMBF und aus dem ESF der EU (Förderkennzeichen 16OH11008, 16OH11009, 16OH11010), in der zweiten Förderphase (2015-2017) aus Mitteln des BMBF (Förderkennzeichen 16OH12008, 16OH12009, 16OH12010) gefördert worden. Weitere Informationen: www. wmhoch3.de.
} 
und Selbststudium, die als zwei Varianten des Umgangs mit Zeit und Raum beim wissenschaftlichen Weiterbildungslernen betrachtet werden.

Konzeptionell schließt der Beitrag an die Überlegungen von Kade und Seitter (1996) zu den „Konstitutionsleistungen“ von Weiterbildungsteilnehmenden beim Zusammenspiel von Teilnahme und Lernpraxis an. ${ }^{6}$ In ihren Analysen arbeiten sie drei Bereiche der Konstitutionsleistungen heraus: „die Herstellung der Bedingungen des Lernens, [...] die Fortsetzung der Teilnahme [...] und die soziale Akzeptanz der Teilnahme“" (1996, S. 157). Für die weiteren Ausführungen sind - aufgrund der Betrachtung von einmaligen Weiterbildungsteilnahmen - die erste und dritte Dimension relevant. Denn wissenschaftliche Weiterbildungsteilnehmende sind - wie die Teilnehmenden der Funkkolleg-Weiterbildungen bei Kade und Seitter - gefordert, Selbstorganisationsleistungen in räumlicher, zeitlicher und auch sozialer Hinsicht zu erbringen (Herstellung der Lernbedingungen) und ihre Lernaktivität „,in den sozialen Raum des Alltags“ (ebd.) zu vermitteln mit allen zugehörigen Aushandlungsprozessen (soziale Akzeptanz).

Zunächst werden Befunde zum Präsenz- und Selbststudium in der wissenschaftlichen Weiterbildung mit Fokus auf zeitlichen und räumlichen Aspekten dargestellt (2). Dann erfolgt ein Überblick zum methodischen Design der „Individuumsbezogenen Zeitbudgetstudie“" und zum Vorgehen bei der Re-Analyse der Lernzeiten und Lernräume (3). Im Anschluss werden die Interviewbefunde zu Lernzeiten und Lernräumen des Präsenzstudiums (4.1) und des Selbststudiums (4.2) dargelegt, miteinander verglichen (4.3) und der Qualitätsaspekt von Lernzeiten und -räumen dargelegt (4.4). Der Schluss resümiert die zeit- und raumbezogenen Ergebnisse zum Präsenz- und Selbststudium wissenschaftlicher Weiterbildung im Kontext von Befunden zu selbstorganisierten Lernprozessen (5).

\section{Befunde zu Präsenz- und Selbstlernphasen in der wissenschaftlichen Weiterbildung}

Ein konstitutives Element der Lernzeit- und Lernortgestaltung wissenschaftlicher Weiterbildungsangebote ist ihre zielgruppenbezogene Konzeption von Präsenz- und Selbststudium. Studien im Bereich wissenschaftlicher Weiterbildung zeigen, dass Teilnehmende flexible Angebote mit kurzen Laufzeiten in zeitlich kompakten Veranstaltungen an Wochenenden präferieren, für die möglichst kurze Wegstrecken zurückzulegen sind (vgl. Kahl et al. 2015, S. 348; Präßler 2015, S. 178f.). Dies spiegelt sich in den vorhandenen Zeitformaten wissenschaftlicher Weiterbildungsangebote und ihrer zeitbezogenen „Distribution im Wochenverlauf“ (Fürst 2017, S. 98) wider. Für 40 weiterbildende Masterstudiengänge und 151 weiterbildende Zertifikatskurse an 16 hessischen Hochschulen ist die Länge und zeitliche Lage von Blockseminaren und Einzelterminen untersucht worden. Es zeigt sich,

\footnotetext{
6 Obgleich die wissenschaftliche Weiterbildung weniger offen und flexibel organisiert ist als das von Kade und Seitter (1996) untersuchte Funkkolleg, profitiert die Analyse der Präsenz- und Selbstlernphasen der wissenschaftlichen Weiterbildung von den konzeptionellen Überlegungen zu den selbstorganisierten Lernprozessen einer Funkkollegteilnahme.
} 
dass das Wochenende in beiden Formaten [Masterstudiengänge und Zertifikatskurse, R.K.] am häufigsten genutzt wird, was gleichzeitig eine hohe Anzahl an zwei- bis dreitätigen Präsenzveranstaltungen impliziert. [...] Einzeltermine werden selten in die Planung von Weiterbildungsangeboten einbezogen (Fürst 2017, S. 114).

Hinsichtlich der Lernorte hat sich herausgestellt, dass „,vorzeigbare Räume“ (Kahl et al. 2015, S. 346) für die kostenpflichtigen Weiterbildungsveranstaltungen erforderlich sind, was je nach Fachdisziplin zur Wahl von hochschulischen oder ausgelagerten Seminarorten führt (vgl. Salland und Rumpf 2017, S. 154).

Die Untersuchung von Habeck und Rundnagel (2017) zu Blockseminaren in der wissenschaftlichen Weiterbildung zeigt, dass die begrenzten zeitlichen Ressourcen der Teilnehmenden die Gestaltung der Lehr-Lernformen maßgeblich mit bedingen. Im Unterschied zum grundständigen Studium werden sie methodisch-didaktisch an die Bedarfe berufstätiger Studierender angepasst, indem sie stärker mit Selbstlernanteilen verbunden sind und vermehrt Blended-Learning-Konzepte zum Einsatz kommen (eine Komination aus E-Learning und Präsenzterminen). Zudem findet eine größere Verzahnung der Lerninhalte zwischen ,,vorbereitende(r) Selbstlern-, Präsenz- oder nachbereitende(r) Selbstlernphase“" (Habeck und Rundnagel 2017, S. 127) statt.

Beim Selbststudium schätzen Teilnehmende die freie Zeitgestaltung und Ortswahl, obgleich diese häufig zu Vereinbarkeitsschwierigkeiten mit dem beruflichen und privaten Umfeld führen (vgl. Wonneberger et al. 2015, S. 78). Die Lernzeitbudgetstudie im Rahmen der „Individuumsbezogenen Zeitbudgetstudie“ hat ergeben, dass der präferierte Lernort für das Selbststudium das Zuhause ist, der Arbeitsplatz, die Hochschule und andere Orte spielen eine nachgelagerte Rolle (vgl. Denninger et al. 2017b, S. 99f.).

\section{Methodisches Vorgehen}

Die Datengrundlage des Beitrags entstammt dem Forschungsprojekt „Individuumsbezogene Zeitbudgetstudie“ (2015-2017). Es handelt sich um eine explorative Mixed-Methods-Untersuchung mit zwei gleichrangigen, komplementär angelegten Teilstudien: einer qualitativen Interviewstudie zur zeitlichen Vereinbarkeit der Weiterbildungsteilnahme und einer quantitativen Zeitprotokollstudie zur Lernzeitverausgabung der Weiterbildungsteilnehmenden (vgl. Abb. 1). Die Stichprobe setzt sich aus Teilnehmenden der Fächergruppen Sozial-, Wirtschafts-, Ingenieurswissenschaften und Medizin ${ }^{7}$ zusammen nach den fachlichen Profilen der drei untersuchten Hochschulen des Verbundprojekts $\mathrm{WM}^{3}$. Die Auswertung des Materials ist zielgruppen-, maßnahmen-, fach- und zeitpunktspezifisch in einer resultatbasierten

\footnotetext{
7 Teilnehmende aus dem Fach Medizin sind lediglich in der zeitlichen Vereinbarkeitsstudie vertreten.
} 
Abb. 1 Forschungsdesign der „Individuumsbezogenen Zeitbudgetstudie" (Denninger et al. 2017a, S. 81 leicht verändert)
Individuumsbezogene Zeitbudgetstudie

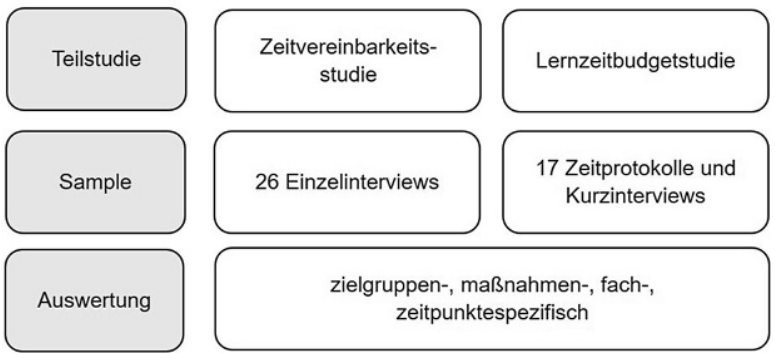

Integration erfolgt, um mögliche Einflüsse dieser Dimensionen auf die Zeitverausgabung und Vereinbarkeit zu berücksichtigen. ${ }^{8}$

In der Teilstudie zur zeitlichen Vereinbarkeit einer wissenschaftlichen Weiterbildungsteilnahme, die für diesen Beitrag als Datengrundlage fungiert, sind insgesamt 26 halbstandardisierte Leitfadeninterviews geführt worden. Mit der gewählten Methode ist die individuelle Perspektive auf die zeitbezogene Studiensituation, deren Bewertung und Strukturmuster in den Blick genommen worden (vgl. Hopf 2005, S. 350). Die sechs Hauptfragebereiche umfassen Teilnahmemotivation, zeitbezogenes Lernverhalten, hinderliche und förderliche Teilnahmefaktoren, Zeitkonkurrenzen zwischen Lebensbereichen, Bewältigungsstrategien und zeitbezogene Zufriedenheit (vgl. Denninger et al. 2017a, S. 83, 2017b, S. 11). Die Auswertung der Interviews ist mit einer computergestützten qualitativen Inhaltsanalyse nach Mayring (2016, S. $114 \mathrm{ff}$.) erfolgt, in der die Hauptthemen als zentrale Kategorien aufgegriffen worden sind.

Bislang ist das Datenmaterial hinsichtlich der temporalen Vereinbarkeit mit verschiedenen Lebensbereichen, der Lernzeitverausgabung wissenschaftlicher Weiterbildung und der Lernzeiträume des Selbststudiums analysiert worden (vgl. Denninger et al. 2017b, 2018, 2019). Im Weiteren steht die Betrachtung der Lernzeiten und Lernräume im Präsenz- und Selbststudium im Fokus. Die Neubetrachtung der Befunde erfolgt anhand des Interviewmaterials, da es - im Unterschied zu den auf das Selbststudium fokussierten Zeitprotokollen - Auskünfte über beide didaktischen Formate liefert. Zudem geben die qualitativen Befunde Einblick in die individuellen Gestaltungsspielräume und Bewertungen von Lernzeiten und Lernräumen.

\section{Lernzeiten und Lernräume wissenschaftlicher Weiterbildung}

Wissenschaftliche Weiterbildungsangebote sind in ihrer didaktischen Gestaltung von Selbstlern- und Präsenzphasen auf die zeitlichen Ressourcen und die räumlichen

\footnotetext{
8 Zielgruppenspezifika: Familienpflichten, berufliche Vorbildung, berufliche Position; Maßnahmenspezifika: Zertifikatskurs, Weiterbildungsmaster, konsekutiv-dualer Masterstudiengang, hochschulisch-postgraduales Weiterbildungsangebot; Zeitpunktspezifika: Studienzeitpunkte (Beginn, Mitte, Ende des Angebots).
} 
Bedarfe der berufstätigen erwachsenen Zielgruppe ausgerichtet. In Präsenzphasen, die zumeist als Blockveranstaltungen organisiert sind, werden Zeitpunkt, Zeitdauer und Lernumgebung durch den Weiterbildungsanbieter hergestellt und festlegt. Bei den Selbstlernphasen sind die Teilnehmenden gefordert, eine eigenständige zeitlichräumliche Organisation des Lernens vorzunehmen. Jede dieser beiden Studienphasen weist für Teilnehmende eigene Herausforderungen und Vereinbarkeitsschwierigkeiten bezüglich der Zeitinvestition und der Lernumgebung auf, die im Folgenden anhand von Interviewmaterial veranschaulicht werden.

\subsection{Lernzeiten und -räume von Präsenzphasen}

Die Weiterbildungsteilnehmenden stellen unterschiedliche Vorzüge und Herausforderungen der durch die Hochschule festgelegten Lernorte und Zeiträume der Präsenzveranstaltung heraus. Ein Vorteil von Präsenzphasen liegt in zeitlicher Hinsicht im Bereich der Platzierung im Jahresverlauf. Wenn die hochschulischen Weiterbildungsanbieter die Veranstaltungstermine frühzeitig bekannt geben, können Zeitfenster für die Weiterbildungsteilnahme reserviert und häusliche Abwesenheiten organisiert werden. Das vereinfacht Absprachen und Vereinbarungen im privaten wie beruflichen Bereich und erhöht die Akzeptanz des sozialen Umfelds für die weiterbildungsbezogene Abwesenheit.

[...] man muss dann sozusagen dahin. Das ist irgendwie von außen vorgegeben mehr oder weniger [...]. Dann ist klar, man ist einfach den Tag irgendwie weg oder in der Zeit, in der das stattfindet (Teilnehmende/r 22, Abs. 47).

Außerdem erleichtert die Festlegung von Lernzeit und Lernort durch den Weiterbildungsanbieter eine eindeutige Abgrenzung zu anderen Tätigkeiten und Verpflichtungen. Die Anwesenheit der Lehrperson(en) und anderen Teilnehmenden unterstützt, sich auf die Weiterbildung vor Ort einzulassen.

Die Seminare sind so ausgelegt, dass das Präsenzzeit ist [...] in der Uni. Und die sind dann ganztägig, [...] was ich persönlich als positiv wahrnehme. Das ist ein anderes Lernen [...] im direkten Kontakt. Und auch weil ich einen festen Termin habe, muss ich dahin, und das erleichtert mir das, als wenn es mir jetzt sozusagen dann eigenverantwortlich überlassen wird (Teilnehmende/r 24, Abs. 15).

Vor allem das verbreitete Format der Blockveranstaltungen reduziert Ablenkungen aus anderen Lebensbereichen, so dass eine Fokussierung auf die Lerninhalte deutlich leichter fällt. Allerdings stellt das zeitlich kompakte Lehr-Lernformat hohe Anforderungen an die Konzentrationsleistung der Teilnehmenden und wird als anstrengend wahrgenommen. Dies kann durch die zeitliche Lage der Veranstaltungen im Tages- und Wochenverlauf bedingt sein. So ist die geistige Leistungsfähigkeit bei Abendveranstaltungen nach einem Arbeitstag oder auch Wochenendseminaren im Anschluss an eine Arbeitswoche tendenziell eingeschränkt. Doch auch die Länge der Kompaktseminare selbst kann in Kombination mit den wissenschaftlichen Lerninhalten ermüdend wirken, ,weil das [...] nicht ohne (ist), gerade wenn man nicht gewöhnt ist, so acht Stunden super konzentriert sich mit teilweise auch anspruchs- 
vollen Themen auseinanderzusetzen " (Teilnehmende/r 7, Abs. 20). Hierin zeigt sich ein Einfluss des eigenen Befindens auf die Lernmöglichkeiten.

Des Weiteren können Blockveranstaltungen Zeitdruck in anderen Lebensbereichen erzeugen, da sie nur wenig Spielraum für Erledigungen im privaten oder beruflichen Bereich lassen. Dadurch entstehen Aufgabenverdichtungen vor und nach den Präsenzterminen.

[...] wenn also drei Tage am Stück Blockseminare sind von früh bis spät, wo ansonsten gar nichts anderes geht, wo man auch nach 9h Seminar sehr ausgelaugt nach Hause kommt, d.h., alles, was in diesen drei Tagen passiert, sowohl [Arbeitgeber] wie auch zu Hause, kann ich gar nichts machen. Da merke ich es ganz stark, naja privat, weil ich ein Totalausfall bin, [Arbeitgeber], weil ich viel vor- und nacharbeiten muss (Teilnehmende/r 1, Abs. 23).

Als nachteilig erweist sich die kompakte Lernzeit an der Hochschule im Besonderen, wenn aufgrund drängender Aufgaben und Verantwortungen - wie Bereitschaftsund Vertretungsdiensten oder der Betreuung von Familienangehörigen - die Anwesenheit nicht möglich ist.

[...] dadurch, dass wir immer so komprimierte Präsenzzeiten haben, würde mir ja wahnsinnig viel fehlen, wenn ich ein ganzes Wochenende sagen würde: „Ich bleibe zu Hause“. Wenn ich in der [Organisation] mal einen Tag nicht da bin, da wird nicht so viel verpasst, aber wenn ich ein ganzes Wochenende nicht zur Uni gehe, weil meine Kinder krank sind, dann geht es ja nicht (Teilnehmende/r 3, Abs. 5).

Eine weitere Herausforderung bei der Teilnahme an Präsenzveranstaltungen resultiert aus dem von der Hochschule festgesetzten Lernort. Abhängig vom Wohnort der Teilnehmenden sind mitunter weite Wegstrecken zur Weiterbildungsstätte zurückzulegen, was zusätzliche temporale Investitionen (für Fahrzeiten und weitere Übernachtungen) und persönliche Belastungen mit sich bringt.

[...] komischerweise stresst mich am meisten die lange Fahrt. Also, ich finde die Seminare spannend, ich finde [Stadt] selber auch interessant und ich finde die Vorlesungen [...] wirklich superinteressant. Aber diese lange Fahrt nervt mich einfach manchmal und das ist halt mit der Bahn einfach/permanent Verspätungen, irgendwelche Ausfälle und man nie so diese Garantie, dass man wirklich pünktlich von A nach B kommt. Und ich glaube, das stresst mich tatsächlich mehr [...] dieses Rumpendeln an und für sich (Teilnehmende/r 11, Abs. 24).

Ein Wechsel des Lehrformats von Präsenzlehre zu E-Learning stellt eine Möglichkeit dar, die Schwierigkeiten der Teilnehmenden hinsichtlich festgelegter Lernzeiten und -orte zu beheben. Doch trotz flexiblerer Gestaltungsmöglichkeiten befürwortet die Mehrheit der Befragten (kompakte) Präsenzveranstaltungen. Die notwendige zeitliche wie räumliche Selbstorganisation und Disziplin beim elektronisch gestützten Lernen erweist sich für viele als belastender Mehraufwand.

Ehrlich gesagt glaube ich, dass die Präsenzzeiten gut sind [...], wenn das E-Learning Phasen wären, dann würde ich die schieben. [...] Aber ich denke 
mir halt, okay, ich bin dann einen Freitag bin ich komplett von neun bis halb sechs in der Uni. Mit Mittagspause und Kaffeepause. Und es ist anstrengend und es ist viel. Aber am Ende des Tages habe ich die zehn Zeitstunden. Und dann muss ich mir keine Gedanken machen, mache ich heute zwei Stunden, mache ich morgen zwei Stunden (Teilnehmende/r 23, Abs. 61).

Ferner erleichtern Präsenzveranstaltungen die Abgrenzung gegenüber dem Arbeitgeber, da eine Notwendigkeit zur Freistellung für die Veranstaltungsteilnahme offenkundig ist.

Die Gefahr [...] wenn ich erzählen würde in meinem Unternehmen, dass ich ja über das Internet mir eine Vorlesung anschauen muss, die geht so und so viel Stunden, dann wäre der erste Kommentar, ja, dann schaue es dir nach der Arbeit an. Und das ist halt ein bisschen schwierig. Wenn ich sage, okay, da kommt ein Professor nach [Stadt] und ich muss anwesend sein, sonst muss ich ein Attest zeigen, dann ist das Fakt. Dann muss ich dahinfahren. Wenn ich mir Ort und Zeit aussuchen kann, dann kann ich mir Ort und Zeit auch aufs Wochenende legen (Teilnehmende/r 16, Abs. 41).

Als weiteres Argument für die Präsenztermine benennen die Befragten den direkten Austausch mit Lehrenden und anderen Teilnehmenden. Das befördert die Aneignung des Lernstoffs, erhält die Weiterbildungsmotivation und ermöglicht den Austausch von Berufserfahrungen und den Aufbau beruflicher Netzwerke unter den Teilnehmenden.

\subsection{Lernzeiten und -räume von Selbstlernphasen}

Die Vorteile und Schwierigkeiten bezüglich der Zeitinvestition und Lernumgebung im Selbststudium liegen vor allem in der selbstorganisierten flexiblen Gestaltung durch die Teilnehmenden begründet. Selbstlernphasen finden am häufigsten Zuhause statt (vgl. Denninger et al. 2017b, S. 99). Das ermöglicht einerseits, sich im privaten Bereich eine passende Lernumgebung einzurichten und jederzeit für die Weiterbildung aktiv zu werden. Allerdings ist die Gefahr der Ablenkung durch das soziale Umfeld und private Verpflichtungen aufgrund der räumlichen Nähe wesentlich höher.

Also ich merke schon, dass das Zuhause dann schwieriger ist, dann kommt ja doch mal derjenige, könntest du nicht mal schnell, kannst du nicht da mitkommen und könntest du mir da mal helfen. Also man lässt sich schon teilweise schnell ablenken (Teilnehmende/r 26, Abs. 19).

Zudem ist die soziale Akzeptanz für Lernzeiten oft eingeschränkter, weil sie von den Teilnehmenden selbst festgelegt werden.

Das Selbststudium obliegt ja so ein bisschen der eigenen Kontrolle und da ist dann vielleicht schon, wenn man am Wochenende mal ein Buch aufklappt, sowas wie: „Na ja, kannst du das nicht wann anders machen?“ Oder da ist dann etwas mehr Frustration, glaube ich, so bei Bekannten, Freunden, Partnerin oder 
wem auch immer als beispielsweise/also irgendwie jetzt als im Vergleich zu den fest vorgegebenen Präsenztagen, ja (Teilnehmende/r 22, Abs. 47).

Neben diesen Faktoren spielt im Selbststudium besonders zu Hause die Selbstdisziplin und -motivation eine entscheidende Rolle. „Es ist schon wirklich wichtig, dass man sich Zeiten reserviert und die auch verteidigt und auch vor sich selber verteidigt, da vielleicht am meisten " (Teilnehmende/r 12, Abs. 91). Feste temporale und räumliche Lernroutinen und -rhythmen im Tages- und Wochenverlauf erweisen sich in diesem Kontext als hilfreich.

Die temporale Lage von Selbstlernphasen im Tagesverlauf und die vorausgegangenen Aktivitäten können sich nachteilig auf die Lernfähigkeit auswirken. Wenn eine mehrstündige Erwerbstätigkeitszeit vorangegangen ist, erleben viele Befragte ihre Leistungsfähigkeit als eingeschränkt. Zudem sind die nachmittäglichen oder abendlichen Lernfortschritte geringer als zu den häufig präferierten Lernzeiten am Vormittag.

Prinzipiell bevorzuge ich Lernen und Arbeiten im Allgemeinen eher so vormittags [...] also tagsüber und weniger jetzt abends, weil ich [...] einfach dann irgendwann mal bemerke nach $17 \mathrm{Uhr}, 18 \mathrm{Uhr}$, $19 \mathrm{Uhr}$ wird es nicht mehr so effizient und man braucht länger. Bleibt nicht mehr so viel hängen. Man ist weniger aufnahmefähig und, ja, es kostet im Endeffekt mehr Zeit, als wenn man das irgendwie über den normalen Tag in normalen Arbeitszeiten sozusagen einbauen kann [...] (Teilnehmende/r 22, Abs. 53).

Eine Möglichkeit, privaten Ablenkungen und abendlichen Konzentrationsschwächen zu entgehen, wären Lernphasen am Arbeitsplatz. Allerdings sind diese nur in Ausnahmefällen umsetzbar, etwa ,wenn es der Beruf am Freitagnachmittag mal zulässt, wenn kein Kunde anruft, dann auch da mal in den Ordner rein(zu)gucken “ (Teilnehmende/r 20, Abs. 5). Selbst wenn sich temporale Gelegenheiten eröffnen, können diese eher selten genutzt werden. Denn der Arbeitsplatz ist mit Erwerbstätigkeit assoziiert; um für die wissenschaftliche Weiterbildung aktiv zu werden, haben einige „(nicht) den Kopf“ (Teilnehmende/r 18, Abs. 13). Außerdem stellt der Arbeitsort eine störanfällige und somit ungeeignete Lernumgebung dar. Die Mehrheit der Befragten beschäftigt sich mit der Weiterbildung ,definitiv nur in der Freizeit zu Hause, wenn man auch mal sagen kann, Telefon zur Seite und auch keiner, der denn da rumläuft und sagt, können wir mal eben hier, können wir mal eben da“ (Teilnehmende/r 19, Abs. 13).

Als begünstigenden Faktor des Selbststudiums benennen einige Befragte das Lernen in Gruppen. Obgleich „,der zeitliche Aufwand [...] der gleiche (ist) “ (Teilnehmende/r 14, Abs. 27) und mögliche Wegstrecken zum Gruppenlernort anfallen, überwiegen die Vorteile durch die kollegiale Unterstützung, gegenseitige Motivation und festgelegten Termine und Treffpunkte.

Andere Lernorte neben dem Zuhause spielen in den Interviews jedoch selten eine Rolle. Im Umfeld von Präsenzphasen fungieren mitunter Hotelzimmer als Lernorte. Außerdem werden Fahrtzeiten mit öffentlichen Verkehrsmitteln erwähnt, ,wie im Zug sitzen oder so, um meinetwegen in ein Buch reinzulesen " (Teilnehmende/r 14, Abs. 47). Solche Orte können kurzfristig als Lernraum konstituiert werden, was 
jedoch selten vorkommt. Denn zwischen den inhaltlichen Aufgaben des Selbststudiums und dem notwendigen Zeitaufwand und Lernort lässt sich ein Zusammenhang feststellen. Das bezieht sich zum einen auf die Dauer einer einzelnen Lerneinheit. Während die Vor- und Nachbereitung von Lehrveranstaltungen, Referatsvorbereitungen oder das Lernen für Klausuren in kürzeren Zeiteinheiten und dadurch auch einmal an öffentlichen Orten erfolgen kann, erfordern ,vor allem so Hausarbeiten [...] eigentlich Zeit am Stück" (Teilnehmende/r 04, Abs. 28), die an den benannten öffentlichen Orten (Hotelzimmer, Verkehrsmittel) meist weniger zur Verfügung steht. ${ }^{9}$

Zum anderen benötigen die verschiedenen Lernanlässe einen unterschiedlich langen Lernzeitraum, was zugleich die Wahl des Zuhauses als Lernraum begünstigt. Komplexe Themen, umfangreicher Lernstoff für Klausuren oder schriftliche Arbeiten erfordern einen kontinuierlichen Zeiteinsatz über mehrere Wochen.

Ich habe dann schon gerade was [Fachgebiet] jetzt eben anbelangt, da wusste ich das wird viel Arbeit. Da habe ich dann schon vor vier, fünf Wochen langsam so angefangen. [...] Bei anderen Sachen jetzt, bei wirklich so AuswendiglernSachen denkst du ja gut, das schaffst du in zwei Wochen [...]. Aber man muss halt für sich wirklich wissen, wie lerne ich (Teilnehmende/r 26, Abs. 53).

Das eigene Lerntempo und geeignete Aneignungsstrategien zu kennen, hilft bei der Planung des zeitlichen Aufwands für Prüfungsleistungen und der Wahl der passenden Lernumgebung. Vor allem Freizeit wird in intensiven Arbeitsphasen für die wissenschaftliche Weiterbildung zur Lernzeit.

Also, es war in der reinen Schreibzeit, [...] von der Hausarbeit [...] da muss man sich schon vor Augen halten, dass da mit Freizeit gar nicht so viel ist. Vielleicht mal ein Abend die Woche, wo man gesagt hat: Okay, heute mache ich mal gar nichts dafür. Aber das war schon so jeden Tag, musste schon irgendwie paar Stunden was gemacht werden (Teilnehmende/r 10, Abs. 9).

In diesem Kontext verwundert es kaum, dass häufig Urlaubszeiten oder Wochenenden genutzt werden. Sie ermöglichen, jenseits beruflicher Verpflichtungen und Beanspruchungen, zu der präferierten Tageszeit zu lernen. Entsprechend zeichnen sich diese Lerneinheiten meist durch eine hohe Leistungs- und Konzentrationsfähigkeit aus. Eine Einschränkung bilden familiäre Verpflichtungen: Wenn Urlaubszeiten zugleich Familienzeiten sind, kann das Lernen ähnlich wie im Berufsalltag in temporale Nischen (spät) abends, nachts oder früh am Morgen verlagert werden.

\subsection{Präsenz- und Selbstlernphasen im Vergleich}

Ob in einem Raum zu einer bestimmten Zeit Lernen für einen wissenschaftlichen Weiterbildungsteilnehmenden stattfinden kann, hängt nach den Befunden mit in-

\footnotetext{
${ }_{9}$ Die Hochschule wird als Lernort meist aufgrund ihrer geographischen Lage nicht genutzt, da die (längere) An- und Abreise für die Befragten einen unerwünschten Mehraufwand darstellt. Besonders bei nichttraditionellen Studierenden lassen sich zudem Hinweise erkennen, dass sie die Hochschule nicht als Lernraum für sich wahrnehmen.
} 
Abb. 2 Individuelle und soziale Einflussfaktoren auf zeitlichräumliche Weiterbildungslernbedingungen (eigene Darstellung)

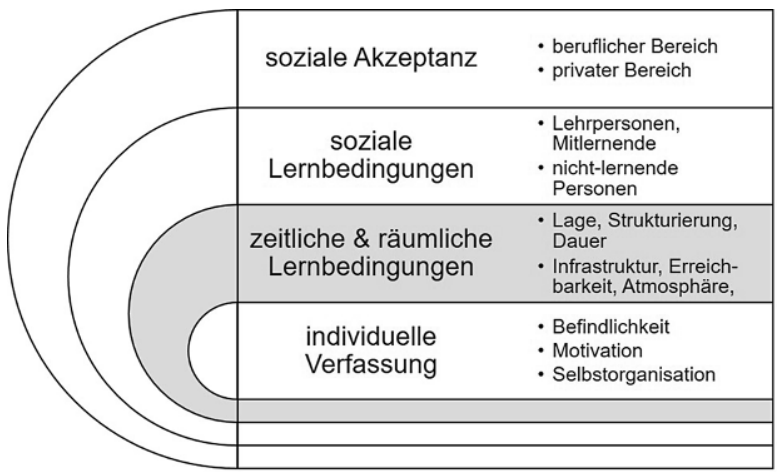

dividuellen Faktoren, temporalen und örtlichen Gegebenheiten und sozialen Kontextbedingungen der Situation zusammen (vgl. Abb. 2). So bedingt die individuelle Verfassung in einer bestimmten Umgebung zu einer bestimmten Zeit, ob in diesem „Zeit-Raum“ lernen möglich ist (Kraus spricht in diesem Zusammenhang von der „Atmosphäre“, 2015, S. 48). Darüber hinaus beeinflusst die An- und Abwesenheit weiterer, am Lernen beteiligter und nicht-beteiligter Personen (Lehrkräfte, Weiterbildungsteilnehmende, Familienmitglieder, Kollegen etc.) und die soziale Akzeptanz der Lerntätigkeit im Lebensumfeld, ob die zeitlichen und räumlichen Bedingungen zum Lernen genutzt werden können (vgl. ebd.; Kade und Seitter 1996, S. 157). ${ }^{10}$

Präsenz- und Selbstlernphasen lassen entlang dieser konzeptionellen Überlegungen folgende unterschiedliche Bedingungsgefüge erkennen:

Für Präsenzphasen ist bedeutsam, dass die zeitliche und räumliche Struktur von den Lehrenden hergestellt wird (im Rahmen der institutionellen Bedingungen) und Materialien, Ausstattung, Infrastruktur und Rhythmisierung des Lernens anbietet. Zudem sind Mitlernende anwesend, während Anforderungen und Ansprachen aus anderen Lebensbereichen weitestgehend reduziert sind. Die meist komprimierten Lehr-Lern-Formate können auf der individuellen Ebene zu einer reduzierten Aufnahmefähigkeit im Tagesverlauf und Lernrückständen bei krankheitsbedingter $\mathrm{Ab}$ wesenheit führen, auf der sozialen Ebene zu Aufgabenverdichtung im beruflichen bzw. privaten Lebensbereich. Dennoch sorgt die zeitlich-räumliche Festlegung durch die hochschulischen Weiterbildungsanbieter für eine hohe Akzeptanz der Präsenzphasen im beruflichen und im - vorrangig davon betroffenen - privaten Umfeld. Von den Teilnehmenden werden sie als verdichtete, didaktisch vorbereitete Weiterbildungslernsettings geschätzt.

Die Selbstlernphasen sind ein maßgeblich selbstorganisierter Lernprozess, für den es Eigenverantwortlichkeit, Zeit- und Raummanagement und eine förderliche geistig-körperliche Verfassung benötigt. Es ist möglich, eigenen temporalen wie örtlichen Lernpräferenzen zu folgen sowie zeitlich-räumliche Gelegenheiten fürs

\footnotetext{
10 Die Konzeption ist anhand der empirischen Befunde unter Berücksichtigung der Konstitutionsleistungen von Weiterbildungsteilnehmenden nach Kade und Seitter (1996, S. 157f.), der Bedingungen für Lernorte nach Kraus (2015, S. 48f.) und der Einflussfaktoren auf Weiterbildungslernzeiten (vgl. Denninger et al. 2017a, S. $65 \mathrm{ff}$.) erfolgt.
} 
Lernen zu nutzen, zugleich ist es voraussetzungsvoller, passende Bedingungen $\mathrm{zu}$ generieren. Vorangegangene berufliche oder private Aktivitäten, eine nicht-präferierte Tageszeit oder mangelnde Infrastruktur können die Lernfähigkeit einschränken. Ablenkungen durch das soziale Umfeld und Konkurrenzen zwischen dem Lernen und anderen Aufgaben begrenzen oder unterbrechen häufig Zeiten und Räume des selbstorganisierten Lernens. In der Regel sind Selbstlernphasen außerhalb der Arbeitszeit anzusiedeln und stellen sich auch im privaten Bereich als begründungsbedürftig dar - besonders, wenn Zuhause gelernt wird. Die soziale Akzeptanz für Selbstlernphasen ist im Weiterbildungsprozess immer wieder herzustellen bzw. einzuholen, da die individuellen Entscheidungen für Lernzeiträume mit dem sozialen Umfeld auszuhandeln sind und es dessen Unterstützung bedarf, damit Lernen stattfinden kann.

Aufgrund der Gestaltungsspielräume bieten die Selbstlernphasen für Weiterbildungsteilnehmende zwar Chancen für eigene zeitlich-räumliche Präferenzsetzungen. Meist erweisen sie sich jedoch als die schwieriger zu bewältigende Lernphase, da sie höhere Organisationskompetenzen in sozialer, zeitlicher und räumlicher Hinsicht erfordern als die von der Hochschule geplanten und strukturierten Präsenzphasen. ${ }^{11}$

\subsection{Qualität von Lernzeiten und Lernorten}

Die benannten förderlichen und hinderlichen Bedingungen bei Selbst- und Präsenzphasen machen deutlich, dass in beiden Studienformen die Qualität der Lernzeit und des Lernorts eine wichtige Rolle spielt. Anhand der Befunde lassen sich insbesondere zwei Bereiche erkennen, für die die Qualität von Zeit und Raum zentral sind:

- die individuelle Dimension der Konzentrationsfähigkeit und

- die angebotsbezogene Dimension der Prüfungsaufgaben.

Die Konzentrationsfähigkeit ist in den Befunden durch persönliche Kapazitäten, flankierende Aktivitäten und Aufgaben sowie soziale Faktoren bedingt. Wie in der betrieblichen und allgemeinen Weiterbildung beeinflussen vorgelagerte Aktivitäten und die Uhrzeit die Leistungsfähigkeit von Lernphasen (vgl. Nahrstedt et al. 1998, S. 19; Schmidt-Lauff 2008, S. 376 ff.). Im Selbststudium vermindern zudem Unterbrechungen und die Verknüpfung von Räumen mit (beruflichen oder privaten) Aufgabenprofilen die Eignung als Weiterbildungslernort. Lernrhythmen ${ }^{12}$, feste Lernorte und selbstorganisierte Gruppentreffen sind individuelle Strategien, die eigene Konzentrationsfähigkeit zu sichern. Weitere Strategien sind intensive Selbstlernphasen an Wochenenden und in Urlaubszeiten, wie sie aus der betrieblichen Weiterbildung bekannt sind (vgl. Schmidt-Lauff 2008, S. 364 ff.). Die Weiterbildungsanbieter generie-

\footnotetext{
11 Insofern stellen E-Learning-Angebote für viele Befragte keine Alternative zu Präsenzphasen dar: Die Lernbedingungen weisen ähnliche herausfordernde Ausprägungen auf wie die Selbstlernphasen; die Vorteile der Präsenzphasen gehen verloren (Lehrende und Mitlernende anwesend, festgelegte Lernorte, -zeiten etc.).

12 Siehe hierzu die analysierten Zeitmuster für Selbstlernphasen in der wissenschaftlichen Weiterbildung (Denninger 2019, S. 117).
} 
ren mit den terminierten Blockphasen und gesonderten Lernorten ihrerseits zeitlichräumliche Bedingungen ,,von Ausschließlichkeit und Ruhe“ (Schmidt-Lauff 2008, S. 386), um die Konzentration der Teilnehmenden zu unterstützen und soziale Ablenkungen zu reduzieren. Damit die geistige Aufnahmefähigkeit über den Seminartag erhalten bleibt, bedarf es einer zielgruppenbezogenen didaktischen Rhythmisierung der Lehre (vgl. Habeck und Rundnagel 2017, S. 126 ff.).

Die Lernaufgaben und Prüfungsleistungen des Weiterbildungsangebots bilden einen zweiten Bereich, für den zeitlich-räumliche Qualitäten bedeutsam sind. Während Veranstaltungsvorbereitungen und -nachbereitungen in kurzen Lerneinheiten und bei Fahrzeiten vorgenommen werden können, erfordern komplexe bzw. umfangreiche Aufgaben wie Klausurvorbereitungen und Hausarbeiten kompakte Selbstlerneinheiten über einen mehrwöchigen Zeitraum. Lernroutinen und Lernrhythmen im Tages-, Wochen- und Modulverlauf erweisen sich ebenso wie selbstorganisierte Lerngruppen als unterstützend. Störanfällige Lernorte oder kurze Zeitfenster sind für solche Aufgabenstellungen weniger geeignet, so dass häufig Wochenenden und Urlaubsphasen genutzt werden. Um ,der stillen Aufwendung primärer und sekundärnachträglicher Zeitanteile für Lernen“" (Schmidt-Lauff 2008, S. 446) in der Weiterbildung entgegenzuwirken und rechtliche wie betriebliche Rahmenbedingungen zu entwickeln, wären zeitliche Spielräume wie durch eine Einführung von Lernzeitkonten zur Flexibilisierung der Lernphasen im Lebenslauf notwendig (vgl. Faulstich 2003, S. 41). Damit könnte ein eigenes Zeitfenster für wissenschaftliche Weiterbildung geschaffen und die Freizeit als primäres Reservoir der Lernzeit entlastet werden.

\section{Fazit}

Zusammengefasst kommen als temporale wie örtliche Verdichtung des Lernens in der wissenschaftlichen Weiterbildung als Präsenzphasen bevorzugt Blockseminare an Wochenenden zum Einsatz, die mit Selbstlernphasen verzahnt sind (vgl. Fürst 2017; Habeck und Rundnagel 2017), welche meist zu Hause nach der Arbeitszeit oder an freien Tagen ausgeübt werden (vgl. Denninger et al. 2017b, S. $91 \mathrm{ff}$.). An Blockveranstaltungen schätzen Teilnehmende, dass sie von der Selbstorganisation und Terminierung der Lerneinheiten entlastet sind und eine externe Legitimierung von Weiterbildungseinheiten gegenüber dem privaten wie beruflichen Umfeld besteht. Das Selbststudium hingegen erfordert eine eigenständige Generierung des Zeitraums und Lernorts, was viele Befragte als Herausforderung hinsichtlich der Selbstdisziplin, des Zeitmanagements und der Aushandlung mit dem sozialen Umfeld erleben (vgl. Wonneberger et al. 2015, S. 78).

Passfähige Lernzeiten und -räume stellen eine wichtige Gelingensbedingung für die Weiterbildungsteilnahme insgesamt dar. Dabei zeigt sich, dass es zeitliche wie räumliche Qualitätsanforderungen an das organisierte Lernen in der wissenschaftlichen Weiterbildung gibt, um „Lernwirksamkeit [...] mit dem Ineinandergreifen von Inhaltlichem und Interaktionellem“ (Tietgens 1982, S. 129) zu ermöglichen. Zeiten und Orte für das Präsenz- und besonders für das Selbststudium sind dabei stets 
sozial zu legitimieren und im individuellen Lebensumfeld zu etablieren. Nicht nur vor der Weiterbildungsteilnahme, sondern insbesondere währenddessen bedarf es der Zustimmung und Unterstützung des Umfelds. Der Erfolg der zeitlichen und räumlichen Etablierungsbemühungen der wissenschaftlichen Weiterbildungsaktivität zwischen Arbeitszeit und privater Zeit hängt von der Flexibilität aller Beteiligten (Anbieter, Teilnehmende und ihre beruflichen und privaten Kontexte) ab (vgl. Kahl et al. 2015, S. 403). ${ }^{13}$ Im Anschluss an Kade und Seitter (1996) lässt sich feststellen, dass zeitliche und räumliche Anforderungen einer wissenschaftlichen Weiterbildungsteilnahme eine Konstitutionsleistung von Seiten der Weiterbildungsanbieter (in der Konzeption des Angebots und Organisation der Präsenzphasen) und der Teilnehmenden (Zeitfenster für Präsenzphasen und Organisation von Selbstlernphasen) darstellen. Wissenschaftliche Weiterbildungsteilnehmende sind ferner gefordert, ,die kontinuierliche Isolierung des Lernens vom Alltagsleben“ (Kade und Seitter 1996, S. 157) bei Präsenz- und Selbstlernphasen sozial zu legitimieren. Anders gesagt liegt es vor allem an den Konstitutionsleistungen der Teilnehmenden, ob wissenschaftliche Weiterbildung zeitweilig als eigener Lebensbereich etabliert werden kann. Insofern erweist sich wissenschaftliches Weiterbildungslernen als eine ,prekäre Lernform“ (Lauber-Pohle 2018, S. 63), die der Lernbefürwortung und Stabilisierung durch das soziale Umfeld und hoher individueller Raum- und ,Zeitkompetenz“ (Schmidt-Lauff 2008, S. 446) bedarf, um Lernzeiten und Lernräume erfolgreich zu etablieren.

Open Access Dieser Artikel wird unter der Creative Commons Namensnennung 4.0 International Lizenz (http://creativecommons.org/licenses/by/4.0/deed.de) veröffentlicht, welche die Nutzung, Vervielfältigung, Bearbeitung, Verbreitung und Wiedergabe in jeglichem Medium und Format erlaubt, sofern Sie den/die ursprünglichen Autor(en) und die Quelle ordnungsgemäß nennen, einen Link zur Creative Commons Lizenz beifügen und angeben, ob Änderungen vorgenommen wurden.

\section{Literatur}

CEDEFOP (2012). Training leave. Policies and practice in Europe. http://www.cedefop.europa.eu/files/ 5528_en.pdf. Zugegriffen: 11. Apr. 2019.

Denninger, A., Kahl, R., \& Döring, A. (2019). Räumliche Lernzeitverausgabung des Selbststudiums in der wissenschaftlichen Weiterbildung. In W. Seitter \& T. Feld (Hrsg.), Räume in der wissenschaftlichen Weiterbildung (S. 100-125). Wiesbaden: Springer.

Denninger, A., Kahl, R., \& Präßler, S. (2017a). Individuumsbezogene Zeitbudgetstudie - Konzeptionen zur Erhebung der Zeitverausgabung von Teilnehmenden wissenschaftlicher Weiterbildung. In W. Seitter (Hrsg.), Zeit in der wissenschaftlichen Weiterbildung (S. 59-93). Wiesbaden: Springer.

Denninger, A., Kahl, R., Präßler, S., \& Döring, A. (2017b). Individuumsbezogene Zeitbudgetstudie. http:// www.wmhoch3.de/images/Individuumsbezogene_Zeitbudgetstudie.pdf. Zugegriffen: 14. Dez. 2018.

Denninger, A., Kahl, R., \& Präßler, S. (2018). Zeitliche Optimierungsbedarfe zur Vereinbarkeit von wissenschaftlicher Weiterbildung und Berufstätigkeit aus Teilnehmendenperspektive. In W. Seitter, M. Friese \& P. Robinson (Hrsg.), Wissenschaftliche Weiterbildung zwischen Implementierung und Optimierung (S. 35-63). Wiesbaden: Springer.

13 Hinsichtlich der Flexibilität und sozialen Legitimierung zeigen sich Parallelen zur betrieblichen Weiterbildung (vgl. Schmidt-Lauff 2008, S. 408) und dem Online-Lernen (vgl. Lauber-Pohle 2018, S. 63). Inwiefern die Befunde zu zeitlichen und räumlichen Qualitäten auf andere Weiterbildungsarten von Erwachsenen zutreffen oder übertragbar sind, bildet ein Forschungsdesiderat. 
Faulstich, P. (2003). Weiterbildung und Arbeitszeit. Begründungen alternativer Zeitstrukturen für Lernchancen. In R. Dobischat, H. Seifert \& E. Ahlene (Hrsg.), Integration von Arbeit und Lernen. Erfahrungen aus der Praxis des Lebenslangen Lernens (S. 17-45). Berlin: Sigma.

Faulstich, P. (2005). Lernzeiten - Zeit zum Lernen öffnen. In G. Wiesner \& A. Wolter (Hrsg.), Die lernende Gesellschaft. Lernkulturen und Kompetenzentwicklung in der Wissensgesellschaft (S. 213-224). Weinheim: Juventa.

Fürst, C. (2017). Zeitformate in der wissenschaftlichen Weiterbildung. In W. Seitter (Hrsg.), Zeit in der wissenschaftlichen Weiterbildung (S. 97-119). Wiesbaden: Springer.

Habeck, S., \& Rundnagel, H. (2017). Blockseminare in der wissenschaftlichen Weiterbildung. In W. Seitter (Hrsg.), Zeit in der wissenschaftlichen Weiterbildung (S. 119-141). Wiesbaden: Springer.

Hanft, A., \& Knust, M. (2010). Berufsbegleitendes Studieren. Bildungspolitisches Anforderungen und internationale Praxis. Zeitschrift für Soziologie der Erziehung und Sozialisation ZSE, 30(1), 43-59.

Hopf, C. (2005). Qualitative Interviews. Ein Überblick. In U. Flick, E. Kardorff \& I. Steinke (Hrsg.), Qualitative Forschung. Ein Handbuch (4. Aufl. S. 349-360). Hamburg: Rowohlt.

Jütte, W., \& Bade-Becker, U. (2018). Weiterbildung an Hochschulen. In R. Tippelt \& A. Hippel (Hrsg.), Handbuch Erwachsenenbildung/Weiterbildung (6. Aufl. S. 821-836). Wiesbaden: Springer.

Kade, J., \& Seitter, W. (1996). Lebenslanges Lernen. Mögliche Bildungswelten. Erwachsenenbildung, Biographie und Alltag. Opladen: Leske \& Budrich.

Kahl, R., Lengler, A., \& Präßler, S. (2015). Akzeptanzanalyse. Forschungsbericht zur Akzeptanz innerhochschulischer Zielgruppen. In W. Seitter, M. Schemmann \& U. Vossebein (Hrsg.), Zielgruppen in der wissenschaftlichen Weiterbildung (S. 291-408). Wiesbaden: Springer.

Kraus, K. (2015). Orte des Lernens als temporäre Konstellationen. Ein Beitrag zur Diskussion des Lernortkonzepts. In C. Bernhard, K. Kraus, S. Schreiber-Barsch \& R. Stang (Hrsg.), Erwachsenenbildung und Raum. Theoretische Perspektiven - professionelles Handeln - Rahmungen des Lernens (S. 41-53). Bielefeld: W. Bertelsmann.

Lauber-Pohle, S. (2018). Soziale Netzwerkbildung und Online-Lernen. Wiesbaden: Springer.

Mayring, P. (2016). Einführung in die qualitative Sozialforschung. Eine Anleitung zu qualitativem Denken (6. Aufl.). Weinheim: Beltz.

Nahrstedt, W., Brinkmann, D., Kadel, V., Kuper, K., \& Schmidt, M. (1998). Neue Zeitfenster für Weiterbildung. Temporale Muster der Angebotsgestaltung und Zeitpräferenzen der Teilnehmer im Wandel. Bielefeld: IFKA.

Präßler, S. (2015). Bedarfserhebung. Forschungsbericht zu Bedarfen individueller Zielgruppen. In W. Seitter, M. Schemmann \& U. Vossebein (Hrsg.), Zielgruppen in der wissenschaftlichen Weiterbildung (S. 61-187). Wiesbaden: Springer.

Rumpf, M., \& Salland, C. (2017). Lehren und Lernen in der wissenschaftlichen Weiterbildung. Eine Fachkulturanalyse. http://www.wmhoch3.de/images/Fachspezifische_Lehr-Lernkulturanalysen.pdf. Zugegriffen: 18. Dez. 2018.

Schmidt-Lauff, S. (2008). Zeit für Bildung im Erwachsenenalter. Interdisziplinäre und empirische Zugänge. Münster, New York, München, Berlin: Waxmann.

Schmidt-Lauff, S. (2012). Grundüberlegungen zu Zeit und Bildung. In S. Schmidt-Lauff (Hrsg.), Zeit und Bildung. Annäherung an eine zeittheoretische Grundlegung (S. 11-60). Münster, New York, München, Berlin: Waxmann.

Schulmeister, R., \& Metzger, C. (2011). Die Workload im Bachelor: Ein empirisches Forschungsprojekt. In R. Schulmeister \& C. Metzger (Hrsg.), Die Workload im Bachelor: Zeitbudget und Studierverhalten. Eine empirische Studie (S. 13-128). Münster: Waxmann.

Seitter, W. (2017a). Wissenschaftliche Weiterbildung. Multiple Verständnisse - hybride Positionierungen. Hessische Blätter für Volksbildung HBV, 67(2), 14-151.

Seitter, W. (2017b). Zeit in der wissenschaftlichen Weiterbildung. Eine Einleitung. In W. Seitter (Hrsg.), Zeit in der wissenschaftlichen Weiterbildung (S. 9-18). Wiesbaden: Springer.

Sommerfeldt, H., \& Höllermann, P. (2014). Trendstudie Fernstudium 2014. Ergebnisse der Fernstudienumfrage $2014 \mathrm{zu}$ aktuellen Trends und Entwicklungen in deutschsprachigen Fernstudienprogrammen. https://www.trendstudie-fernstudium.de/s/Trendstudie-Fernstudium-2014-Hohe-Qualitat.pdf. Zugegriffen: 18. Dez. 2018.

Stang, R., Bernhard, C., Kraus, K., \& Schreiber-Barsch, S. (2018). Lernräume in der Erwachsenenbildung. In R. Tippelt \& A. Hippel (Hrsg.), Handbuch Erwachsenenbildung/Weiterbildung (6. Aufl. S. 643-658). Wiesbaden: Springer.

Thuy, P., \& Höllermann, P. (2011). Trendstudie Fernstudium 2011. Aktuelle Trends und Entwicklungen in Fernstudienprogrammen der Betriebswirtschaftslehre in Deutschland. https://static1.squarespace. 
com/static/57286de5e707ebe0b4240685/t/5729aac6b6aa60bb6d5d2d2a/1472047025775/2011++Trendstudie+Fernstudium+2011.pdf. Zugegriffen: 18. Dez. 2018.

Tietgens, H. (1982). Angebotsplanung. In E. Nuissl (Hrsg.), Taschenbuch der Erwachsenenbildung (S. 122-144). Baltmannsweiler: Burgbücherei Schneider.

Vogel, B., \& Woisch, A. (2013). Orte des Selbststudiums. Eine empirische Studie zur zeitlichen und räumlichen Organisation des Lernens von Studierenden. http://www.dzhw.eu/pdf/pub_fh/fh-201307.pdf. Zugegriffen: 18. Dez. 2018.

Wonneberger, A., Weidtmann, K., Hoffmann, K., \& Draheim, S. (2015). Die Öffnung von Hochschulen durch flexible Studienformate am Beispiel zweier neuer weiterbildender Masterstudiengänge. Beiträge zur Hochschulforschung BZH, 37(1), 70-91.

Publisher's Note Springer Nature remains neutral with regard to jurisdictional claims in published maps and institutional affiliations. 